\title{
The Importance of Mediator Training in Applied Behavior Analysis
}

\author{
Dimitra Chaldi \\ Correspondence: Dimitra Chaldi, PhD. Student, M.ADS, BSc. S-LP. School of Medicine, University of Patra, 26504, \\ Patra, Greece
}

\author{
Received: April 24, 2021 Accepted: February 2, 2022 Online Published: February 13, 2022 \\ doi:10.5539/res.v14n1p22 URL: https://doi.org/10.5539/res.v14n1p22
}

\begin{abstract}
The mediator training is an important procedure of the applied behavior analysis. By providing an appropriate training to the mediator, the trainer can enhance the likelihood that the trainee will implement the intervention plan accurately. The purpose of this study was to operate mediator training to an instructor therapist to implement mand training to a 5-year old girl with autism spectrum disorder. Through this study we had to follow all the necessary steps of the mediator training and to collect data, through the behavior fidelity checklist and the staff satisfaction survey. The results demonstrated that the mediator implemented the steps accurately and she was also satisfied from the overall training that she received. Moreover, the interobserver agreement for the treatment fidelity, between the comparison of trainers and trainee's checklist, demonstrated exact agreement of the implementation of the intervention.
\end{abstract}

Keywords: mediator training, mand training, fidelity checklist, consumer satisfaction, interobserver agreement

\section{Introduction}

Training is an important strategy and part of the Applied Behavior Analysis (ABA), for modifying the behavior and the skills of mediators (e.g., staff, parents, teachers, etc.). Through training, behavior analysts (BAs) will work with the mediators to give them the necessary skills that they need, to be part of their behavior support plan. Main goal of the mediator training is to enhance mediators' attitude, effectiveness, technical expertise, and their knowledge based on the specific behavior-change plan (Bailey \& Burch, 2010, pp. 275-284). For our case, we need to provide training to our mediator, who will be a new-hired instructor therapist (IT) and who will work two days a week with our client. Her training will be focused on how she can implement assessment and measurement procedures, and after that how she can provide mand training to Maria; a 5-year-old girl who was diagnosed with autism spectrum disorder (ASD), engages in challenging behavior(s), and lacks a vocal repertoire. Initially, we conducted a literature review, to examine research papers about mediator training in staff population in general, and then studies, which were focused on how we can train staff to implement mand intervention. Through these studies, we will be able to examine what are the best practices and steps that we can follow for our mediator training. Providing well-trained staff is a key to ensuring good quality of autism services. Based on Dillenburger, McKerr, Jordan, and Keenan (2016), individuals with ASD need higher support, compared with other populations, in terms of daily living, and in their physical and mental health. Their study identified that poorly-trained staff (any kind of professionals) were unable to implement the interventions appropriately and accurately, and at the same time their bad quality of training, increased their level of stress and anxiety, due to their lack to support those people. This study, recommended that it should be provided better quality of staff training which should be based on international best practice. By improving training procedure, it will also improve individuals' with ASD quality of life, and staff will be less likely to face emotional stress. Another study showed that a 3-day staff training, can highly increase staffs' confidence who were working with individuals with ASD and aggressive behaviors. After the training, they could manage aggressive behaviors and feel more confident during the implementation of the intervention strategies. Nevertheless, the study demonstrated that there was not any evidence of reduction of clients' challenging behavior. The analysis of this study showed that the training was effective only for the staff and their levels of confidence on handling aggressive behaviors (McDonnell et al., 2008). Based on Jahr (1998), staffs' priority is to improve individuals with developmental disabilities quality of life. That can be achieved through training. His study demonstrated the great importance of staff training and at the same time the procedures which should be followed to achieve an effective training. He also stated that it is important, except of enhancing staffs' skills, to maintain these skills, and transfer them across variety of settings, programs, and clients. He stated that prompt-fading techniques, establishing teaching techniques to improve those peoples' skills, and transferring these skills across all the clients' programs, should be part of their training plan. At the end, he suggested that further study needed it to improve training across variety of behavioral techniques, assessment, and measurement procedures. That can be achieved by conducting training by having smaller scale, more precise definitions, and variables. In our study, we will conduct staff training for developing mand requesting to our client. 
Madzharova, Sturmey, and Jones (2012) conducted a study which was separated in two case studies. On the first one, they provided training to peer-to-peer mand and evaluated the full package of training (instruction, modeling, rehearsal, and feedback). On the second study, they conducted only modeling and feedback. Through their results, they identified that in both training cases, staff increased correct teaching responses, and at the same time individuals increased the number of independent peer-to-peer mands. The only limitation of this study was that it was not considered as an experiment, so, researchers suggested the development of studies which will use experimental designs, and not case studies, and will replicate the results. After the analysis of the articles above, we identified the importance of staff training. More specifically, all of them agreed that staff training can increase staffs' confidence in the implementation of the intervention plans, and reduce staffs' levels of anxiety and stress. Moreover, they agreed that staff training, can enhance individuals with developmental disabilities skills, by following the specific steps of the training, and our final article (Madzaharova et al., 2012) argued that staff training can effectively increase the independent manding to children with ASD. Based on these articles, we can highly recommend the implementation of staff training for Maria, to develop her mands which at the same time will lead to the decrease of her challenging behavior (i.e., screaming).

\section{Participant}

Mari is a 5-year-old girl who was diagnosed with ASD, engages in challenging behaviors, and lacks a vocal repertoire. Thus, every time she wants to have access to preferred items, she engages in screaming behavior. Maria lives with her parents and has a typically developing adolescent sister. Based on the Verbal Behavior Milestones Assessment and Placement Program (VB-MAPP) (Sundberd, 2008), Maria has strong play, echoic and imitation skills, and she can also follow simple one-step instructions. The areas most in need of further development are an increase in requesting skills listener responder skills, expressive language skills, visual perceptual skills, matching-to-sample, and finally a decrease in problematic behavior including screaming, flopping, and head-banging (screaming is precursor for the occurrence of the last two behaviors).

\section{Operational Definition}

Screaming is defined as a vocalization above conversational level for at least 2, and loud enough for us to hear across a room, which ends when there are no vocalizations for 5 seconds. Screaming maybe a precursor for crying, hitting, flopping, and/or head-banging. Non-examples include whispering or speaking at a conversational level with whole words or vocal approximations. Manding (requesting) is defined as appropriately and with a low voice tone, asking for things that our client wants when there is motivation for it. Non-examples include screaming, hitting, and crying to have access to preferred item.

\subsection{Social Relevance of the Behavior Change}

Maria's challenging behavior pose a barrier to her learning, limit her social interaction opportunities, and my lead to restrictive placements in the future (e.g., residential settings, institutions, etc.) (Koegel, L.K., Koegel, R.L., Boettcher, Harroer, \& Openden, 2006). Replacing her challenging behavior with appropriate communication will increase the likelihood of optimal outcomes in these areas, and of course on her quality of life (e.g., develop and maintain friendships, decrease the danger for the occurrence of health problems due to her head banging, etc.).

\section{Description of the Mediator (i.e., Instructor Therapist)}

Maria's team had two instructor therapists (ITs) so far and she used to attend 2 days a week Intensive Behavior Intervention (IBI). Recently she received funding, thus she will attend the center 5-days a week. For that reason, a new-hired IT, who will be our mediator, will be part of Maria's team. The mediator is a young girl who recently graduated from the psychology department and she had minimum experience of working with children with ASD, and more specifically with children who also engaged in challenging behavior(s), like Maria. Nevertheless, she has a lot of knowledge on ASD, but she lacks experience. She has no professional certification in behavior analysis and has not previously received training to manding. Although, she had previous training in behavior analysis and was familiar with behavioral analytic language. For that reason, we will train her how to teach Maria manding (requesting) for preferred items, without engaging in challenging behavior(s) (e.g., screaming). Our expectation from her, will be to be able to implement all the necessary intervention steps accurately, and record, graph, and analyze the data.

\subsection{Consent Process for Mediator}

Before starting the mediator training, mediator provided written consent. Based on that form, we informed the mediator what the mediator training will include, what Maria's intervention will include, the importance of the accurate implementation of the strategy and recording all the data, and the importance of keeping our client's information anonymous. The form also included the potential risks, and benefits of the intervention plan and all the steps that will be followed during the mediator training. 


\section{Method}

One of the most important skill set for every behavior therapist (BT) is to learn how to conduct the behavior skill training (BST). BST is an evidence-based training method which has been used effectively to train individuals, parents, and staff to implement intervention for individuals with developmental disabilities (DD) (Parsons, Rollyson, \& Reid, 2012; Buck, 2014). Based on Buck (2014), BST is an effective training package which includes the following components: instructions, modeling, rehearsal, and feedback. Conducting BST is a skill that all BTs should acquire to be able to train mediators to effectively provide intervention to support staff, parents, teachers and other support personnel. Parsons et al. (2012) stated that if mediators are not effectively trained, they will be unable to implement behavior change intervention. There are two main features to BST, the performance and the competency-based training components. The performance component involves the trainer (BT), and mediator/trainee (e.g., staff), whereby trainer models the target skill, and then the mediator will role-play the target skills. The competency component involves training the mediator until they can competently perform the target skills. For example, the mediator would be expected to achieve a competency score of 90 $-100 \%$ as determined by the performance checklist created by the BT (Parsons et al., 2012). The BST protocol consists of 6 steps initially, but was increased to 8 steps (Parsons et al., 2012, 2013) which were designed for staff training in a group format; however, it can be adapted for individual training as well. In step 1, the trainer will provide the rationale for the skill being trained. The target skill/behavior (TB) will be operationally defined in measurable and observable terms, and a performance checklist will be developed to measure the staff performing the skills. In Step 2, the trainer will provide vocal description of the skill to be taught, and then a brief written description of how the target skill and the components involved will be provided to the mediators. In Step $3 \& 4$, the BT/trainer will demonstrate the skill to the mediator, modelling the process. The rehearsal/role-play is step 5, where the mediator practices the taught skill with another staff or the BC. This is the most important component in the BST, because it must be performed correctly to enable effective implementation of the intervention. In Step 6 the trainer will take data on the correct/incorrect performance during role-play teaching the target skill. For step 7, the trainer will provide both corrective feedback and social praise to the mediator. The eighth step is the final step, and this involves the mediator(s), repeating steps 5 to 7 until the performance criterion set is achieved (Parsons et al., 2012, 2013).

\subsection{Description of Instructions (Mediator Skill to Be Taught)}

The following antecedent manipulations will be incorporated into the intervention package. First, Maria will receive functional communication training (FCT)/mand training, which will encourage her to request for preferred item(s). Maria will also receive paired-choice stimuli preference assessment, which will be conducted prior to her sessions, to identify which reinforcers Maria is motivated for in a day, and at the same time to prevent the occurrence of screaming.

\subsection{FCT/Mand Training}

Mediator will teach Maria how she can appropriately ask for item(s), when the reinforcer is present, with whole word or an appropriate verbal approximation. Through this skill, the mediator should expect that Maria will independently mand for item(s) when motivation is present using either a whole word or an appropriate verbal approximation. That skill should be taught in natural environment training (NET), and the opportunities to facilitate requesting, should occur naturally. The mediator should also make sure that Maria is motivated for the item. If she does not, mediator should not teach her to request for this item. Instead she can move to something else and try to contrive motivation the next time. Mediator can gain Maria's motivation, either by presenting the item in front of her and play/engaging with it, either when Maria has the item, remove it with a countdown, and as she is motivated for the item, we can prompt her to request for it. The mediator should be aware and when teaching this skill try to do not speak before (What do you want?), otherwise Maria will become responder rather than an initiator. The exception to this will be only if Maria is on prompt steps. The mediator should also try to teach her 2-4 mands at a time and at the same time do several trials back to back.

\subsection{Steps for FTC}

The mediator initially should contrive motivation for an item, by giving Maria a freebie. Once mediator has established Maria's motivation, she can prompt vocally, by saying the name of the item. She should follow this step for several trials until Maria begins to echo the mediator right away or at the same time with her. Once this occurs, mediator begins by giving only partial prompts. The prompting procedure that she should use has most to least hierarchy, by starting with full verbal, then partial, and lastly independent. The mediator should fade prompts systematically based on mastery criteria. If Maria errors and does not make the correct request or does not respond within 3 seconds, the mediator should provide verbal prompt. If Maria echoes her request, mediator should provide the item immediately. The last step will be to set up similar situations and create motivating operation (MO) for the same item, by providing less prompting. After every correct response (FR1) mediator should reinforce Maria's request, by giving her the item, which is the true reinforcer and at the same time verbal praise. Once Maria has mastered this step, mediator should help Maria to generalize that skill, by implementing it across novel locations, people, and stimuli. The final step will be to maintain that skill and work on that 
initially weekly, then bi-weekly, and finally monthly. The mediator across all her sessions should collect data (trial by trial), and then she should graph her data on the daily percentage of correct mands, and record on skill tracking form. The mastery criterion for Maria will be to engage to appropriate requesting (for each step and stimuli) $100 \%$ for 2 consecutive days or $80 \%$ for 3 consecutive days across 2 ITs.

\subsection{Steps for Preference Assessment}

The mediator should also conduct preference assessment prior to Maria's session, to see which reinforcers Maria is motivated for in a day, and to prevent the occurrence of her challenging behavior. The mediator will choose items, which Maria interacts more within a day, more specifically she will choose 8 items, which will write them on the data sheet. Then she will let Maria sample of each item. If the item is edible, the sample should be small ( 1 skittle). If the item is a toy, let Maria interact with it around $30 \mathrm{secs}-1 \mathrm{~min}$. Place in pairs the items in front of Maria and tell her pick one. As soon as Maria grabs the one, mediator should fast remove the other item, and record the data on the data sheet. Mediator should do that for all the 8 items, where one time they will be presented on the left side, and the other time on the right side.

\subsection{Performance Criterion}

Support staff must achieve a $100 \%$ on the performance checklist. The reason for this is because each step listed is a critical component to ensure the efficacy of the intervention. Based on Weck, Bohn, Ginzburg, and Stangier (2011), the treatment integrity can provide important information about to what extent the intervention strategies have been implemented.

\subsection{Social Validity}

Social validity is the acceptability and satisfaction of the intervention procedures, which is usually assessed by soliciting opinions from the individuals who received/implemented the procedure/intervention. To determine the social validly of the intervention package, a consumer satisfaction checklist will be made about mediators' opinion of the training, the intervention package and its impact on Maria's life. Furthermore, a social validity survey will be completed by the support staff.

\section{Results}

Part of our training was during the observation to record data for our mediator's performance. Thus, on our performance checklist we recorded the steps that she or she did not follow. The overall score for mediator's performance was $100 \%$ accurate implementation of the mand program and following all the necessary steps. We had 10 steps that we observed during mand program, and the mediator implemented accurately all of them. In overall, we are very satisfied from our mediator performance.

\subsection{Consumer Satisfaction}

At the end of our training we asked from the mediator to fill in the mediator survey, to determine if she was or not satisfied from her training. The survey covered 11 parts, in which the mediator answered to all of them that she was satisfied (agreed). More specifically the overall score of agree was $100 \%$.

\subsection{Knowledge Test}

We did not have to implement knowledge test, as our mediator had already knowledge about ASD and mand training. Her knowledge was very good. The only thing that she wanted was practice more. For that case, we did not have to include any knowledge test.

\subsection{Trainer BST Fidelity Checklist}

During our training to make sure that we are following all the necessary steps that we should follow during that procedure, we created a fidelity checklist, for the trainer. The results showed that the trainer, $100 \%$ implemented all the steps, with accuracy and appropriately, based on Parsons et al. (2013). After the analysis of all these data, and by observing our client working with the mediator, we can say that, the training was very effective, supportive, and gave the chance to our trainee to enhance her knowledge and improve her behavior skills.

\subsection{Interobserver Agreement (IOA)}

For the IOA, the trainee had to complete a checklist for the treatment fidelity and check all the steps that completed during the intervention. The trainer filled the same checklist as she was observing the trainee during the implementation of the intervention to our client. The analysis of both checklists showed that there was an exact agreement between the two checklists with $100 \%$ agreement.

\section{Discussion}

Even if the training and the feedback that we received from the trainee were very supportive and positive, there are few technical things that we should have in mind for the next time that we will provide training. We should think about our 
training's limitation and try to improve them for the next time. One technical improvement would be the role-play step with our trainee. Unfortunately, due to lack of time we did not have the chance to run couple of times this step. Based on Parsons et al. $(2012,2013)$, the role-play/rehearsal step is very important and should pay a lot of attention, as it helps trainer to identify if the trainee can demonstrate appropriately the skill, and at the same time, is very helpful for the trainee to do an unofficial practice of the skills before the implementation. That will make her feel more confident and sure. Another technical improvement would be to spend more time in our training procedure in overall. Based again on Parsons et al. (2012, 2013), and based on Dillenburger et al (2016) mediator training is a very important step, as providing a careful training, and more time on that, enhance the chances that we will have a well-trained trainee, who will guarantee a good quality of treatment implementation. Fast implementation of the training, may be risky and we might have the opposite results, as we can be always lucky. We need to pay more attention on that. One last technical improvement would be to do not have during my training my client with me at the same time. I had to discontinue my training couple of times. Thus, due to lack of time, I had to observe both my client and trainee at the same time. That probably did not give me the chance to be more focus and provide more support to my trainee. Even if she was satisfied in overall, I feel that it was a limitation to do two things at the same time and it was also very stressful and overwhelming. Our studies limitation, all of them not spending too much time during the whole intervention, and especially during role-play, and the implementing the training when at the same time we had to observe another client, occurred due to lack of time in our center. Unfortunately, that period the center is very busy with many children and all the STs had clients and meetings, and at the same time the trainer did not have free time to do the training, as she had to do the training when at the same time she had to look for another client. This is considered as the biggest and the most important limitation of our study, which for the next time could be avoided, by organizing better the time and the schedule of the trainer. It might be easier to have an hour gap, free time, every time she wants to do the training, to do not be in rush. By asking from the center and the supervisor more time to do the training appropriately, we might mitigate all the limitation above. Even if the study had few limitations and gaps, there were also few strengths of the project. The knowledge that our trainee had before the training, it was very helpful and gave us a breath, as we did not have to spend more time on that. And the trainee seems that had a lot of knowledge and she was very positive to be trained and she was very collaborative which helps us to the training smoothly. Also, even if we had all these limitations, we had at least the fidelity checklist to make sure that the trainee follows all the steps, so at least one person (trainer) spend time to carefully observe her, and seems that our training was very effective, considering the limitations and the lack of time, as the trainee did all the steps, and based on her consumer satisfaction survey seems that she was very satisfied from her overall teaching.To mitigate any limitation, we should also arrange a long-term IOA plan, where we can say that every two days we will observer our mediator and we collect data with our checklists and compare it with her checklist, and other days we can have two observers who will observe the mediator. In that case, we will make sure that the trainer consistently provides the intervention appropriately.

\section{Conclusion}

The mediator training is a procedure on which every BA should pay a lot of attention. It is not a process which can be completed within few hours and then expecting that the trainee will be able to appropriately implement all the steps. through personal study I had the chance to learn many things about training and it seems very easy, put when it came the time to introduce all these steps to another behavior therapist, we realize that we should be very responsible and try to spend as much time as need it until you know that the trainee will independently implement the intervention without making any mistake. Through this study, we realized that we need to spend more time in our training and try consistently to observe our trainee if we can to ensure our client's progress and at the same time our trainee's satisfaction and improvement in the field.

\section{References}

Bailey, J. S., \& Burch, M. R. (2010). Training, Coaching, and Mentoring. In Bailey, J. S., \& Burch, M. R. (Eds.), 25 Essential Skills \& Strategies for the Professional Behavior Analyst (pp. 275- 284). New York, NY: Routledge. https://doi.org/10.4324/9780203879184-24

Bailey, J. S., \& Burch, M. R. (2016). Assessing Behavior (Code 3.0). In Bailey, J. S., \& Burch, M. R. (Eds.), Ethics for behavior analysts: 3rd Edition (pp. 109-122). New York, NY: Routelge. https://doi.org/10.4324/9781315669212

Buck, H. M. (2014). The efficacy of behavior skills training: A literature review (master's thesis). Retrieved from http://opensiuc.lib.siu.edu/cgi/viewcontent.cgi?article=1728\&context=gs_rp.

Dillenburger, K., McKerr, L., Jordan, J. A., \& Keenan, M. (2016). Staff Training in Autism: The One-Eyed Wo/Man...International Journal of Environmental Research and Public Health, 13(7), 1-17. https://doi.org/10.3390/ijerph13070716

Jahr, E. (1998). Current issues in staff training. Research in Developmental Disabilities, 19(1), $73-87$. https://doi.org/10.1016/S0891-4222(97)00030-9 
Koegel, L. K., Koegel, R. L., Boettcher, M. A., Harrower, J., \& Openden, D. (2006). Combining functional assessment and self-management procedures to rapidly reduce disruptive behaviors. In Koegel, R. L., \& Koegel, L. K. (Eds.), Pivotal Response Treatments for Autism (pp. 245-258). Baltimore, Maryland: Paul H. Brooks Publishing Co., Inc.

Madzaharova, M. S., Sturmey, P., \& Jones, E. A. (2012). Training staff to increase manding in students with autism: Two preliminary case studies. Behavioral Interventions, 27(4), 224-235. https://doi.org/10.1002/bin.1349

McDonnell, A., Sturmey, P., Oliver, C., Cunningham, J., Hayes, S., Galvin, M., ... Cunningham, C. (2008). Research in Autism Spectrum Disorders, 2(2), 311-319. https://doi.org/10.1016/j.rasd.2007.08.001

Sundberg, M. L. (2008). VB-MAPP: Verbal behavior milestones assessment and placement program. Concord: AVB Press.

Weck, F., Bohn, C., Ginzburg, D. M., \& Stangier, U. (2011). Assessment of adherence and competence in cognitive therapy: comparing session segments with entire sessions. Psychotherapy Research, 21(6), 658-669. https://doi.org/10.1080/10503307.2011.602751

\section{Copyrights}

Copyright for this article is retained by the author(s), with first publication rights granted to the journal.

This is an open-access article distributed under the terms and conditions of the Creative Commons Attribution license (http://creativecommons.org/licenses/by/4.0/). 